\title{
A case of non validity of the MRTD concept
}

\author{
Krzysztof Chrzanowski ${ }^{1,2}$ \\ ${ }^{1}$ Military University of Technology, Institute of Optoelectronics, 00-908 Warsaw, Poland \\ ${ }^{2}$ Inframet, Graniczna 24, Kwirynow, 05-082 Stare Babice, Poland
}

Received November 10, 2012; accepted December 16, 2012; published December 31, 2012

\begin{abstract}
MRTD is considered as the most important parameter of thermal imagers. It is a function of a minimum temperature difference between the bars of the standard 4-bar target and the background required to resolve the thermal image of the bars by an observer versus spatial frequency of the target. Both theoretical models and experimental tests presented in available literature show MRTD as a monotonically increasing bijective mathematical function. This paper presents the results of measurements of MRTD that contradict this common opinion. These results show that in rare cases MRTD can be a non-bijective function. Imagers having such an MRTD function can fulfill formal requirements on MRTD but will perform poorly in real life conditions.
\end{abstract}

Thermal cameras are imaging systems used to enhance human ability to see in darkness and poor visibility conditions. MRTD is a measure of ability of a thermal imager to detect and recognize targets on a non-uniform background. In detail, MRTD is a subjective parameter that describes the ability of an imager-human system for the detection of low contrast details of an observed object. It is a function of the minimum temperature difference between the bars of the standard 4-bar target and the background required to resolve the thermal image of the bars by an observer versus the spatial frequency of the target.

Both theoretical models and experimental tests presented in available literature show MRTD as a monotonically increasing bijective mathematical function [1-7] (Fig. 1). It is considered as normal situation that higher temperature differences enable to recognize a four bar pattern of higher spatial frequency (smaller bar pattern).

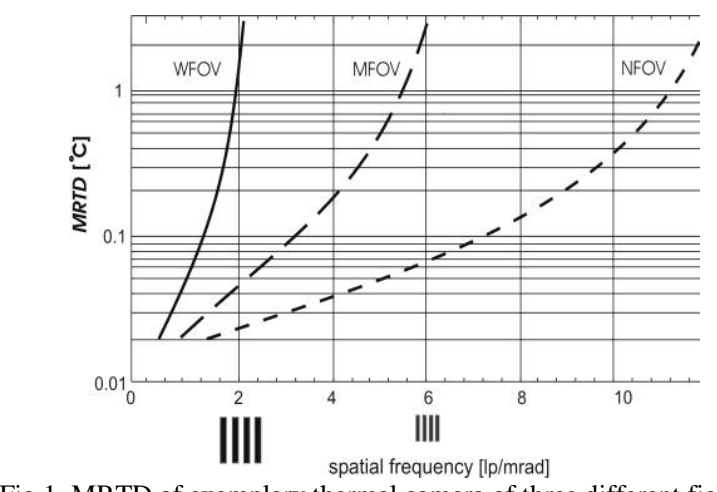

Fig.1. MRTD of exemplary thermal camera of three different field of view.
MRTD is considered as the most important parameter of thermal imagers due to recommendations of international standards [1-2]. Technical specifications of many tenders world wide use requirements on MRTD of surveillance thermal imagers in the form similar to the one presented in Table 1 [8-9].

Table 1. Exemplary requirements on MRTD.

\begin{tabular}{|l|l|l|}
\hline Spatial frequency & MRTD & Comments \\
\hline $1.6 \mathrm{lp} / \mathrm{mrad}$ & $<0.08^{\circ} \mathrm{C}$ & for wide FOV \\
\hline $4 \mathrm{lp} / \mathrm{mrad}$ & $<0.18^{\circ} \mathrm{C}$ & for medium FOV \\
\hline $7.2 \mathrm{lp} / \mathrm{mrad}$ & $<0.16^{\circ} \mathrm{C}$ & for narrow FOV \\
\hline
\end{tabular}

MRTD was criticized in several publications due to low accuracy of measurements resulting from subjective character, low accuracy of performance modeling over Nyquist frequency [6]. However, the general concept of MRTD has always been found properly working. Lower values of MRTD at a specified spatial frequency mean better MRTD. Next, better MRTD means better performance of a tested thermal imager under real field conditions.

The author of this paper has carried out tests of many thermal imagers and his professional experience supports a typical opinion that MRTD is a good parameter to characterize the performance of surveillance thermal imagers. However, recent tests of several thermal imagers offered on the international market showed surprising results. There can be met thermal imagers having MRTD in the form of a non-bijective function shown in Fig. 2.

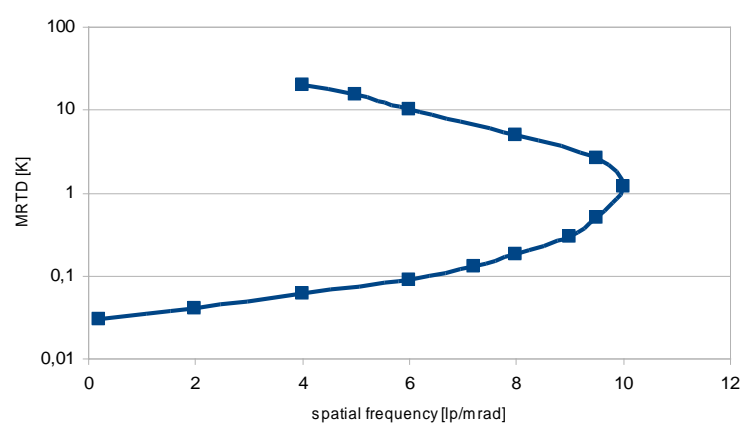

Fig. 2. Measured MRTD in the form of a non-bijective function. 
The tested imager of the MRTD shown in Fig. 2 formally fulfilled the requirement of a tender for which the author was engaged: "MRTD to be below $0.16 \mathrm{~K}$ at $7.2 \mathrm{lp} / \mathrm{mrad}$ ". However, practically there are serious doubts about the performance of the tested thermal imager in real field conditions.

The MRTD graph shown in Fig. 2 practically means that the tested imager will deliver sharp images of targets of temperature difference relative to ambient temperature below about $1 \mathrm{~K}$. However, generated images will become blurred when the temperature difference is higher than $1 \mathrm{~K}$. In other words, higher target temperature difference means that the tested imager will generate more blurred images.

This situation is graphically presented in Fig. 3. We can see that for a temperature difference equal to $0.25 \mathrm{~K}$ it is possible to recognize a four-bar pattern but the image is totally blurred for a temperature difference equal to $7 \mathrm{~K}$.

a)

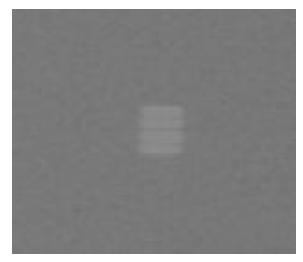

b)

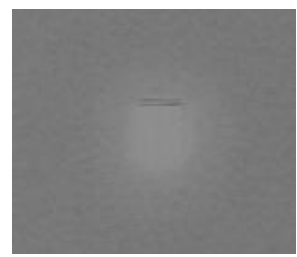

Fig. 3. Image of the same 4-bar target of $7.2 \mathrm{lp} / \mathrm{mrad}$ spatial frequency for two temperature differences: a) $0.25 \mathrm{~K}$, b) $7 \mathrm{~K}$.

The majority of potential targets for surveillance thermal imagers are targets of a temperature difference over a level of $1 \mathrm{~K}$. Therefore the tested imager is poorly suited for real life applications in spite of the fact that the imager fulfills the tender requirement on the MRTD function.

a)

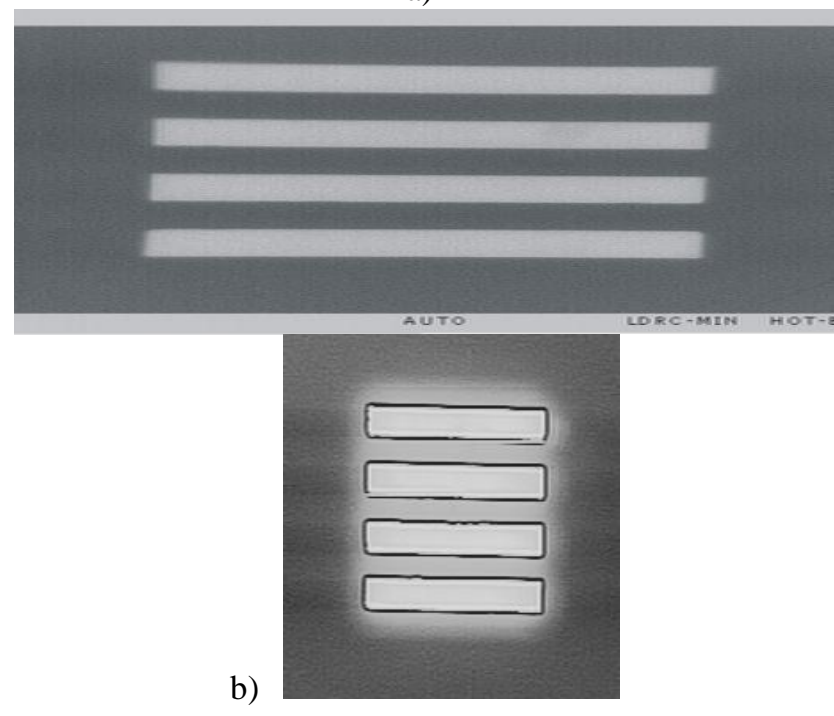

Fig.4. Image of the 4-bar target at two temperature differences: a) $1 \mathrm{~K}$, b) $15 \mathrm{~K}$.

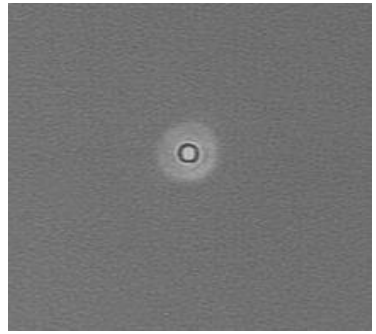

Fig.5. Image of a pinhole target of temperature difference equal to $15 \mathrm{~K}$.

As can be seen in Figs. 4-5, the tested imagers generate sharp, low noise images at targets of low temperature difference but the images of high temperature targets are not only blurred but, additionally, artificial black rectangle patterns are added.

It should be emphasized that the results presented in Figs. 2-5 were obtained while testing thermal imagers run using a manufacturer computer program and at the settings recommended by the manufacturer.

No details about sensor electronics and image processing were disclosed by the manufacturer of the tested thermal imagers. Therefore the author does not know for sure what the reason is for this exceptional behavior of the tested thermal imagers. The most probable source is highly non-linear electronics that does the processing of signals from IR FPA sensor. However, it is not clear if the described situation is a result of an error in the manufacturing technology or if it is a result of a calculated policy of the manufacturer to improve MRTD measurement results at all costs in order to fulfill the formal requirements of a tender.

The manufacturer's name and imager type cannot be disclosed due to test contract restrictions. It can be said only that the tested imagers were built using cooled staring IR FPA sensors.

To summarize, this paper should be treated as a warning for the international community of specialists testing thermal imagers. The message is that on the market one can rarely meet cooled thermal imagers, based on staring IR FPA technology, of potentially very good MRTD fulfilling typical tender requirements but of poor erformance in real life conditions. Therefore it can be recommended that a requirement be added that thermal imagers having non-bijective MRTD functions should not be accepted.

\section{References}

[1] STANAG 4349, Measurement of minimum resolvable thermal difference (MRTD) of thermal cameras, 1995.

[2] ASTM standard E 1213-2002, Standard Test Method for Minimum Resolvable Temperature Difference for Thermal Imaging Systems

[3] G.C. Holst, Testing and evaluation of infrared imaging systems (JCD Publishing Company 2008). 
[4] G.C. Holst, The Infrared \& Electro-Optical Systems Handbook, Vol.3: Electro-Optical System Design, Analysis and Testing, ch. 4, 206-207 (SPIE 1993).

[5] K. Chrzanowski, Testing Thermal Imagers - Practical Guide (Military University of Technology, Warsaw, Poland 2010).

[6] Night Vision Thermal Imaging Systems Performance Model, User's Manual \& Reference Guide, U.S Army Night Vision and Electronic Sensors Directorate, AMSEL-RD-NV-MS-SPMD, 2001.

[7] R.G. Driggers, A. Van Hodgkin, R.H. Vollmerhausen, P. O'Shea, Proc. SPIE 5076, 179 (2003).

[8] http://www.merx.com/English/supplier menu.asp?WCE=Show\&TAB $=1 \&$ PORTAL $=$ MERX\&State=7\&id=PW-\%24QCV-00110993\&FED ONLY=0\&hcode=MoMbi3zoYWn9aYQhn7AEQA\%3 D\%3D

[9] Phare Program no PL0103.03.01 PL0108.19.01-L002, Purchase and installation of portable thermo-vision cameras, Phare Program no PL0103.03.02 purchase of 5 surveillance units for helicopters, 2004. 